\title{
The equivalence between the convergence of the modified Mann and Ishikawa iterations for asymptotically pseudocontractive mappings obtained by dropping the bounded assumption
}

Guiwen Lv ${ }^{*}$ and Zhiqun Xue

*Correspondence:

Ivguiwenyy@126.com

Department of Mathematics and

Physics, Shijiazhuang Tiedao

University, Shijiazhuang, 050043, P.R. China

\begin{abstract}
In this paper, we show the equivalence of convergence between the modified Mann and Ishikawa iterations with errors for an asymptotically pseudocontractive mapping under the condition of removing the bounded assumption. We also point out the problems of (Rhoades and Soltuz in J. Math. Anal. Appl. 283:681-688, 2003; Xue in Bull. Korean Math. Soc. 47(2):295-305, 2010; Xue in J. Math. Inequal. 4(3):345-354, 2010), extend and improve the results of (Zeng in Acta Math. Sin. 47(2):219-228, 2004).

MSC: $47 \mathrm{H} 10 ; 47 \mathrm{H09}$; 46B20
\end{abstract}

Keywords: fixed point; asymptotically pseudocontractive mappings; modified Mann iteration with errors; Banach spaces

\section{Introduction}

Let $E$ be a real Banach space and $E^{*}$ be its dual space. The normalized duality mapping $J: E \rightarrow 2^{E^{*}}$ is defined by

$$
J(x)=\left\{f \in E^{*}:\langle x, f\rangle=\|x\|^{2}=\|f\|^{2}\right\}, \quad \forall x \in E,
$$

where $\langle\cdot, \cdot\rangle$ denotes the generalized duality pairing. The single-valued normalized duality mapping is denoted by $j$.

Let $D$ be a nonempty closed convex subset of $E$ and $T: D \rightarrow D$ be a mapping.

Definition 1.1 [1]

(1) $T$ is called asymptotically nonexpansive if there exists a sequence $\left\{k_{n}\right\} \subset[1,+\infty)$ with $\lim _{n \rightarrow \infty} k_{n}=1$ such that for all $x, y \in D$,

$$
\left\|T^{n} x-T^{n} y\right\| \leq k_{n}\|x-y\|, \quad \forall n \geq 1
$$

(2) $T$ is called asymptotically pseudocontractive if there exists a sequence 
$\left\{k_{n}\right\} \subset[1,+\infty)$ with $\lim _{n \rightarrow \infty} k_{n}=1$ such that for all $x, y \in D$, there exists

$j(x-y) \in J(x-y)$,

$$
\left\langle T^{n} x-T^{n} y, j(x-y)\right\rangle \leq k_{n}\|x-y\|^{2}, \quad \forall n \geq 1 .
$$

Remark 1.2 [2] It is very well known that the following conditions are equivalent:

(i) $T$ is an asymptotically pseudocontractive map;

(ii) there exists $k_{n} \subset[1,+\infty)$ with $\lim _{n \rightarrow \infty} k_{n}=1$ such that

$$
\|x-y\| \leq\left\|x-y+r\left[\left(k_{n} I-T^{n}\right) x-\left(k_{n} I-T^{n}\right) y\right]\right\|, \quad \forall x, y \in D, \forall r>0 .
$$

Definition 1.3 A mapping $T$ is called uniformly $L$-Lipschitzs if there exists $L>0$ such that for any $x, y \in D$,

$$
\left\|T^{n} x-T^{n} y\right\| \leq L\|x-y\|, \quad \forall n \geq 1
$$

Obviously, the asymptotically pseudocontractive and asymptotically nonexpansive mappings with the constant sequence $\{1\}$ are the usual definition of strongly pseudocontractive and nonexpansive mappings, respectively. An asymptotically nonexpansive mapping is asymptotically pseudocontractive. The converse is not true in general; see [3]. And it is clear that an asymptotically nonexpansive mapping is also uniformly $L$-Lipschitz for some $L \geq 1$, where $L=\sup _{n \geq 1}\left\{k_{n}\right\}$.

Let us recall some iterations in the following.

Definition 1.4 For arbitrary given $x_{1} \in D$, the modified Ishikawa iteration with errors $\left\{x_{n}\right\}_{n=1}^{\infty}$ is defined by

$$
\left\{\begin{array}{l}
y_{n}=\left(1-\beta_{n}-\delta_{n}\right) x_{n}+\beta_{n} T^{n} x_{n}+\delta_{n} v_{n}, \\
x_{n+1}=\left(1-\alpha_{n}-\gamma_{n}\right) x_{n}+\alpha_{n} T^{n} y_{n}+\gamma_{n} u_{n}, \quad \forall n \geq 1,
\end{array}\right.
$$

where $\left\{u_{n}\right\},\left\{v_{n}\right\}$ are any bounded sequences of $D .\left\{\alpha_{n}\right\},\left\{\beta_{n}\right\},\left\{\gamma_{n}\right\},\left\{\delta_{n}\right\}$ are four real sequences in $[0,1]$ satisfying $\alpha_{n}+\gamma_{n} \leq 1$ and $\beta_{n}+\delta_{n} \leq 1$ for any $n \geq 1$.

If $\beta_{n}=\delta_{n}=0$ for all $n \geq 1$, then (1.2) reduces to the modified Mann iteration with errors $\left\{z_{n}\right\}_{n=1}^{\infty}$ as follows:

$$
z_{n+1}=\left(1-\alpha_{n}-\gamma_{n}\right) z_{n}+\alpha_{n} T^{n} z_{n}+\gamma_{n} w_{n}, \quad \forall n \geq 1
$$

If $\gamma_{n}=\delta_{n}=0$ for any $n \geq 1$, then for $x_{1}, z_{1} \in D,(1.2)$ and (1.3) reduce to the modified Ishikawa and Mann iterations as follows, respectively (see [4] and [5]):

$$
\begin{aligned}
& \left\{\begin{array}{l}
y_{n}=\left(1-\beta_{n}\right) x_{n}+\beta_{n} T^{n} x_{n}, \\
x_{n+1}=\left(1-\alpha_{n}\right) x_{n}+\alpha_{n} T^{n} y_{n}, \quad \forall n \geq 1,
\end{array}\right. \\
& z_{n+1}=\left(1-\alpha_{n}\right) z_{n}+\alpha_{n} T^{n} z_{n}, \quad \forall n \geq 1 .
\end{aligned}
$$


Recently, many authors [2, 6-8] have proved the iterative approximation problem of fixed point for uniformly $L$-Lipschitz asymptotically pseudocontractive mappings in Banach spaces. The results are as follows.

Theorem 1.5 ([6], Theorem 2.1) Let E be a real Banach space, $D$ be a nonempty closed convex subset of $E$ and $T_{1}: D \rightarrow D$ be a uniformly $L_{1}$-Lipschitzian asymptotically $\Phi$ pseudocontractive mapping with the sequence $\left\{k_{1 n}\right\} \subset[0,+\infty), \lim _{n \rightarrow \infty} k_{1 n}=1$. Let $q \in$ $F\left(T_{1}\right)=\left\{x \in D, T_{1} x=x\right\}$. Let $\left\{a_{n}\right\},\left\{c_{n}\right\} \subset[0,1]$ and satisfy $\sum_{n=1}^{\infty} a_{n}=\infty, \lim _{n \rightarrow \infty} a_{n}=0$ and $c_{n}=o\left(a_{n}\right)$ with $a_{n}+c_{n} \leq 1$ for all $n \geq 1$. Then the Mann iterative process with errors $\left\{u_{n}\right\}$ defined by

$$
\left\{\begin{array}{l}
\forall u_{1} \in D \\
u_{n+1}=\left(1-a_{n}-c_{n}\right) u_{n}+a_{n} T_{1}^{n} u_{n}+c_{n} v_{n}, \quad \forall n \geq 1
\end{array}\right.
$$

converges strongly to $q$.

Theorem 1.6 ([6], Theorem 2.2) Let E be a real Banach space, $D$ be a nonempty closed convex subset of $E$ and $T_{i}: D \rightarrow D(i=1,2)$ be two uniformly L-Lipschitzian asymptotically $\Phi$-pseudocontractive mappings with the sequences $\left\{k_{1 n}\right\},\left\{k_{2 n}\right\} \subset[0,+\infty)$ such that $\lim _{n \rightarrow \infty} k_{1 n}=\lim _{n \rightarrow \infty} k_{2 n}=1$ and $F\left(T_{1}\right) \cap F\left(T_{2}\right)=\emptyset$. Let $\left\{a_{n}\right\},\left\{b_{n}\right\}$ be two sequences in $[0,1]$ satisfying the conditions: (i) $\lim _{n \rightarrow \infty} a_{n}=\lim _{n \rightarrow \infty} b_{n}=0$; (ii) $\sum_{n=1}^{\infty} a_{n}=\infty$. Then the following two assertions are equivalent:

(i) the Mann iteration with errors $\left\{u_{n}\right\}$ converges strongly to the fixed point of $T_{1}$;

(ii) the Ishikawa iteration with errors $\left\{x_{n}\right\}$ converges strongly to the fixed point of $T_{1} \cap T_{2}$.

Remark 1.7 There exists a gap in the proof process of Theorem 2.1 of [6]. It is in lines 3-4 of P300, 'inf ${ }_{n \geq N} \frac{\Phi\left(\left\|u_{n+1}-q\right\|\right)}{1+\left\|u_{n+1}-q\right\|^{2}}=0 \Rightarrow \lim _{j \rightarrow \infty}\left\|u_{n_{j}+1}-q\right\|=0$ ', where $\left\{u_{n_{j}}-q\right\}$ is an infinite subsequence of the sequence $\left\{u_{n}-q\right\}$. Meanwhile, there exists a similar problem in Theorem 2.2 of [6] (for more details, see 11th of P303). For this, we provide an example. Let $\Phi(t)=t, t \in[0,+\infty),\left\|u_{n+1}-q\right\|=n$, then $\inf _{n \geq N} \frac{\Phi\left(\left\|u_{n+1}-q\right\|\right)}{1+\left\|u_{n+1}-q\right\|^{2}}=\inf _{n \geq N} \frac{n}{1+n^{2}}=0$, but there does not exist any subsequence $\left\{n_{j}\right\}$ of the sequence $\{n\}$ such that $\lim _{j \rightarrow \infty} n_{j}=0$. Hence we cannot obtain that $\forall \varepsilon>0, \forall m \in N,\left\|u_{n_{j}+m}-q\right\|<\varepsilon$. So Theorems 2.1, 2.2 of [6] do not hold.

Theorem 1.8 ([2], Theorem 8) Let X be a real Banach space, B be a nonempty closed convex subset of $X$ and $\left\{x_{n}\right\},\left\{z_{n}\right\}$ be defined by (1.5) and (1.4) with $\left\{\alpha_{n}\right\},\left\{\beta_{n}\right\}$ satisfying the following conditions: $\lim _{n \rightarrow \infty} \alpha_{n}=0, \lim _{n \rightarrow \infty} \beta_{n}=0, \sum_{n=1}^{\infty} \alpha_{n}=\infty$. Let $T$ be an asymptotically pseudocontractive and uniformly L-Lipschitzian with $L \geq 1$ self-map of $B$. Let $x^{*}$ be the fixed point of $T$. If $x_{0}=z_{0} \in B$, then the following two assertions are equivalent:

(i) the modified Mann iteration (1.5) converges to $x^{*} \in F(T)$;

(ii) the modified Ishikawa iteration (1.4) converges to $x^{*} \in F(T)$.

But there exists an error in the proof course for the above theorem, i.e., P684 the following formula

$$
\left\|\left(1+\alpha_{n}^{2}\right)\left(x_{n+1}-z_{n+1}\right)+\alpha_{n}\left(\left(\alpha_{n} k_{n} I-T^{n}\right) x_{n+1}-\left(\alpha_{n} k_{n} I-T^{n}\right) z_{n+1}\right) \geq\left(1+\alpha_{n}^{2}\right)\right\| x_{n+1}-z_{n+1} \|
$$


does not hold. The reason is $\lim _{n \rightarrow \infty} \alpha_{n} k_{n}=0 \neq 1$. By remark (1.2), the result of [2] does not hold.

In 2004, Zeng [8] gave another result as follows.

Theorem 1.9 ([8], Theorem 3.1) Let E be a real Banach space, $D$ be a nonempty closed convex subset of $E$ and $T: D \rightarrow D$ be a uniformly L-Lipschitzian asymptotically pseudocontractive mapping with the sequence $\left\{k_{n}\right\} \subset[0,+\infty), \lim _{n \rightarrow \infty} k_{n}=1$. Suppose that $\left\{x_{n}\right\}$ is defined by (1.2), where $\left\{\alpha_{n}\right\},\left\{\gamma_{n}\right\},\left\{\beta_{n}\right\},\left\{\delta_{n}\right\}$ are four real number sequences in $[0,1]$ satisfying the following conditions:

(i) $\alpha_{n}+\gamma_{n} \leq 1, \beta_{n}+\delta_{n} \leq 1$;

(ii) $\lim _{n \rightarrow \infty} \alpha_{n}=0, \sum_{n=0}^{\infty} \alpha_{n}=\infty$;

(iii) $\sum_{n=0}^{\infty} \alpha_{n}^{2}<\infty, \sum_{n=0}^{\infty} \gamma_{n}<\infty$;

(iv) $\sum_{n=0}^{\infty} \alpha_{n}\left(\beta_{n}+\delta_{n}\right)<\infty, \sum_{n=0}^{\infty} \alpha_{n}\left(k_{n}-1\right)<\infty$.

Suppose that the range of $T$ is bounded and $q \in F(T) \neq \emptyset$. If there exists a strictly increasing continuous function $\Phi:[0,+\infty) \rightarrow[0,+\infty)$ with $\Phi(0)=0$ such that

$$
\left\langle T^{n} x_{n+1}-q, j\left(x_{n+1}-q\right)\right\rangle \leq k_{n}\left\|x_{n+1}-q\right\|^{2}-\Phi\left(\left\|x_{n+1}-q\right\|\right), \quad \forall n \geq 0,
$$

then the modified Ishikawa iteration with errors $\left\{x_{n}\right\}$ converges strongly to $q \in F(T)$.

But this result is not perfect because of the assumption of bounded range.

The aim of this paper is to revise the results of the papers [2, 6, 7] and remove the assumption $T$ with bounded range [8]. We obtain that the modified Ishikawa iteration with errors converges strongly to the fixed point of $T$ and the modified Mann and Ishikawa iterations with errors are equivalent. For these, we need the following lemmas.

Lemma 1.10 [9] Let E be a real Banach space and let $J: E \rightarrow 2^{E^{*}}$ be a normalized duality mapping. Then

$$
\|x+y\|^{2} \leq\|x\|^{2}+2\langle y, j(x+y)\rangle, \quad \forall j(x+y) \in J(x+y)
$$

for all $x, y \in E$.

Lemma 1.11 [10] Let $\left\{a_{n}\right\},\left\{b_{n}\right\},\left\{c_{n}\right\}$ be three nonnegative real sequences satisfying the inequality

$$
a_{n+1} \leq\left(1+c_{n}\right) a_{n}+b_{n}, \quad n \geq 1 .
$$

If $\sum_{n=0}^{\infty} c_{n}<\infty, \sum_{n=0}^{\infty} b_{n}<\infty$, then $\lim _{n \rightarrow \infty} a_{n}$ exists.

Lemma 1.12 Let $\left\{\theta_{n}\right\},\left\{b_{n}\right\},\left\{c_{n}\right\},\left\{d_{n}\right\},\left\{e_{n}\right\}$ and $\left\{t_{n}\right\}$ be six nonnegative real sequences satisfying the following conditions:

(i) $\lim _{n \rightarrow \infty} t_{n}=0$;

(ii) $\sum_{n=0}^{\infty} t_{n}=\infty$;

(iii) $c_{n}=o\left(t_{n}\right), e_{n}=o\left(t_{n}\right)$;

(iv) $\sum_{n=0}^{\infty} b_{n}<\infty, \sum_{n=0}^{\infty} d_{n}<\infty$. 
Let $\Phi:[0,+\infty) \rightarrow[0,+\infty)$ be a strictly increasing and continuous function with $\Phi(0)=0$ such that

$$
\theta_{n+1}^{2} \leq\left(1+b_{n}+c_{n}\right) \theta_{n}^{2}-t_{n} \sigma\left(\theta_{n+1}\right)+d_{n}+e_{n}, \quad n \geq 0
$$

where $\sigma(t)=\frac{\Phi(t)}{1+\Phi(t)+t^{2}}$. If $\lim _{n \rightarrow \infty} \theta_{n}$ exists, then $\theta_{n} \rightarrow 0$ as $n \rightarrow \infty$.

Proof Since $\lim _{n \rightarrow \infty} \theta_{n}$ exists, we define $M=\sup _{n} \theta_{n}+1$ and $\lim _{n \rightarrow \infty} \theta_{n}=\delta$. We declare that $\delta=0$. If it is not this case, then $\delta>0$, there exists a natural number $N_{1}$ such that $\theta_{n}>\frac{\delta}{2}$ for $n>N_{1}$. Since $\Phi$ is strictly increasing, then $\sigma\left(\theta_{n+1}\right)>\frac{\Phi\left(\frac{\delta}{2}\right)}{1+\Phi(M)+M^{2}}$. From condition (iii), we obtain that there exists $N_{2}>N_{1}$ such that $c_{n}<\frac{1}{4} \frac{\Phi\left(\frac{\delta}{2}\right)}{\left[1+\Phi(M)+M^{2}\right] M^{2}} t_{n}, e_{n}<\frac{1}{4} \frac{\Phi\left(\frac{\delta}{2}\right)}{1+\Phi(M)+M^{2}} t_{n}$ for $n>N_{2}$. By (1.8), we have

$$
\theta_{n+1}^{2} \leq \theta_{n}^{2}-\frac{1}{2} \frac{\Phi\left(\frac{\delta}{2}\right)}{1+\Phi(M)+M^{2}} t_{n}+d_{n}+M^{2} b_{n}, \quad n \geq N_{2}
$$

which implies that

$$
\frac{1}{2} \frac{\Phi\left(\frac{\delta}{2}\right)}{1+\Phi(M)+M^{2}} t_{n} \leq \theta_{n}^{2}-\theta_{n+1}^{2}+d_{n}+M^{2} b_{n}
$$

It leads to

$$
\begin{aligned}
\frac{1}{2} \frac{\Phi\left(\frac{\delta}{2}\right)}{1+\Phi(M)+M^{2}} \sum_{k=N_{2}}^{n} t_{k} & \leq \theta_{N_{2}}^{2}-\theta_{n+1}^{2}+\sum_{k=N_{2}}^{n} d_{k}+M^{2} \sum_{k=N_{2}}^{n} b_{k} \\
& \leq \theta_{N_{2}}^{2}+\sum_{k=N_{2}}^{n} d_{k}+M^{2} \sum_{k=N_{2}}^{n} b_{k} .
\end{aligned}
$$

From (iv) and (1.11), we have $\sum_{n=0}^{\infty} t_{n}<\infty$ which is a contradiction to condition (ii) and so $\delta=0$, i.e., $\lim _{n \rightarrow \infty} \theta_{n}=0$.

\section{Main results}

Theorem 2.1 Let D be a nonempty closed convex subset of the real Banach space E. Suppose that $T: D \longrightarrow D$ is a uniformly L-Lipschitz asymptotically pseudocontractive mapping with the real number sequence $\left\{k_{n}\right\} \subset[1,+\infty), \lim _{n \rightarrow \infty} k_{n}=1$. Let $\left\{x_{n}\right\}$ and $\left\{z_{n}\right\}$ be defined by (1.2) and (1.3), respectively, where $\left\{\alpha_{n}\right\},\left\{\beta_{n}\right\},\left\{\gamma_{n}\right\}$ and $\left\{\delta_{n}\right\}$ are four real number sequences in $[0,1]$ satisfying the following conditions:

(i) $\alpha_{n}+\gamma_{n} \leq 1, \beta_{n}+\delta_{n} \leq 1$;

(ii) $\lim _{n \rightarrow \infty} \alpha_{n}=0, \sum_{n=0}^{\infty} \alpha_{n}=\infty$;

(iii) $\sum_{n=0}^{\infty} \alpha_{n}^{2}<\infty, \sum_{n=0}^{\infty} \gamma_{n}<\infty$;

(iv) $\sum_{n=0}^{\infty} \alpha_{n}\left(\beta_{n}+\delta_{n}\right)<\infty, \sum_{n=0}^{\infty} \alpha_{n}\left(k_{n}-1\right)<\infty$.

Suppose $q \in F(T) \neq \emptyset$. If there exists a strictly increasing continuous function $\Phi:[0,+\infty) \rightarrow$ $[0,+\infty)$ with $\Phi(0)=0$ such that

$$
\left\langle T^{n} x_{n+1}-T^{n} z_{n+1}, j\left(x_{n+1}-z_{n+1}\right)\right\rangle \leq k_{n}\left\|x_{n+1}-z_{n+1}\right\|^{2}-\sigma\left(\left\|x_{n+1}-z_{n+1}\right\|\right), \quad \forall n \geq 0,
$$

where $\sigma(t)=\frac{\Phi(t)}{1+\Phi(t)+t^{2}}$, then the following two assertions are equivalent: 
(1) the modified Mann iteration with errors $\left\{z_{n}\right\}$ converges strongly to $q \in F(T)$;

(2) the modified Ishikawa iteration with errors $\left\{x_{n}\right\}$ converges strongly to $q \in F(T)$.

Proof If the modified Ishikawa iteration with errors sequence $\left\{x_{n}\right\}$ defined by (1.2) converges strongly to $q$, then setting $\beta_{n}=\delta_{n}=0, \forall n \in \mathbf{N}$, we obtain the convergence of the modified Mann iteration with errors sequence $\left\{z_{n}\right\}$ defined by (1.3). Conversely, we only prove that $(1) \Rightarrow(2)$.

Since $\lim _{n \rightarrow \infty}\left\|z_{n}-q\right\|=0, \lim _{n \rightarrow \infty} k_{n}=1$, then $\left\{z_{n}-q\right\},\left\{k_{n}\right\}$ are bounded. Set $M=$ $\max \left\{\sup _{n}\left\|z_{n}-q\right\|, \sup _{n}\left\{k_{n}+1\right\}, \sup _{n}\left\|u_{n}-q\right\|, \sup _{n}\left\|v_{n}-q\right\|, \sup _{n}\left\|w_{n}-q\right\|\right\}$.

First we prove that the sequence $\left\{x_{n}-z_{n}\right\}$ is bounded.

From (1.2) and (1.3), we have

$$
\begin{aligned}
x_{n}= & x_{n+1}+\left(\alpha_{n}+\gamma_{n}\right) x_{n}-\alpha_{n} T^{n} y_{n}-\gamma_{n} u_{n} \\
= & \left(1+\alpha_{n}\right) x_{n+1}+\alpha_{n}\left(k_{n} I-T^{n}\right) x_{n+1}-\left(1+k_{n}\right) \alpha_{n} x_{n+1} \\
& +\left(\alpha_{n}+\gamma_{n}\right) x_{n}+\alpha_{n}\left(T^{n} x_{n+1}-T^{n} y_{n}\right)-\gamma_{n} u_{n} \\
= & \left(1+\alpha_{n}\right) x_{n+1}+\alpha_{n}\left(k_{n} I-T^{n}\right) x_{n+1} \\
& -\left(1+k_{n}\right) \alpha_{n}\left[\left(1-\alpha_{n}-\gamma_{n}\right) x_{n}+\alpha_{n} T^{n} y_{n}+\gamma_{n} u_{n}\right] \\
& +\left(\alpha_{n}+\gamma_{n}\right) x_{n}+\alpha_{n}\left(T^{n} x_{n+1}-T^{n} y_{n}\right)-\gamma_{n} u_{n} \\
= & \left(1+\alpha_{n}\right) x_{n+1}+\alpha_{n}\left(k_{n} I-T^{n}\right) x_{n+1}-k_{n} \alpha_{n} x_{n}+\left(1+k_{n}\right) \alpha_{n} \gamma_{n} x_{n}+\gamma_{n} x_{n} \\
& -\left(1+k_{n}\right) \alpha_{n}^{2}\left(T^{n} y_{n}-x_{n}\right)+\alpha_{n}\left(T^{n} x_{n+1}-T^{n} y_{n}\right)-\left(1+k_{n}\right) \alpha_{n} \gamma_{n} u_{n}-\gamma_{n} u_{n},
\end{aligned}
$$

and

$$
\begin{aligned}
z_{n}= & \left(1+\alpha_{n}\right) z_{n+1}+\alpha_{n}\left(k_{n} I-T^{n}\right) z_{n+1}-k_{n} \alpha_{n} z_{n}+\left(1+k_{n}\right) \alpha_{n} \gamma_{n} z_{n}+\gamma_{n} z_{n} \\
& -\left(1+k_{n}\right) \alpha_{n}^{2}\left(T^{n} z_{n}-z_{n}\right)+\alpha_{n}\left(T^{n} z_{n+1}-T^{n} z_{n}\right)-\left(1+k_{n}\right) \alpha_{n} \gamma_{n} w_{n}-\gamma_{n} w_{n} .
\end{aligned}
$$

Using (2.2) and (2.3), we have

$$
\begin{aligned}
x_{n}-z_{n}= & \left(1+\alpha_{n}\right)\left(x_{n+1}-z_{n+1}\right)+\alpha_{n}\left(k_{n} I-T^{n}\right)\left(x_{n+1}-z_{n+1}\right)-k_{n} \alpha_{n}\left(x_{n}-z_{n}\right) \\
& +\left(1+k_{n}\right) \alpha_{n} \gamma_{n}\left(x_{n}-z_{n}\right)+\gamma_{n}\left(x_{n}-z_{n}\right)+\left(1+k_{n}\right) \alpha_{n}^{2}\left(x_{n}-z_{n}-T^{n} z_{n}+T^{n} y_{n}\right) \\
& +\alpha_{n}\left(T^{n} x_{n+1}-T^{n} y_{n}-T^{n} z_{n+1}+T^{n} z_{n}\right)-\left(1+k_{n}\right) \alpha_{n} \gamma_{n}\left(u_{n}-w_{n}\right)-\gamma_{n}\left(u_{n}-w_{n}\right) .
\end{aligned}
$$

Since $T$ satisfies (2.1), so $T$ is an asymptotically pseudocontractive map. Applying (1.1), we get

$$
\begin{aligned}
& \left\|x_{n}-z_{n}\right\| \\
& \begin{aligned}
\geq & \left(1+\alpha_{n}\right)\left\|\left(x_{n+1}-z_{n+1}\right)+\frac{\alpha_{n}}{1+\alpha_{n}}\left(k_{n} I-T^{n}\right)\left(x_{n+1}-z_{n+1}\right)\right\| \\
& -\left[k_{n} \alpha_{n}+\left(1+k_{n}\right) \alpha_{n} \gamma_{n}+\gamma_{n}\right]\left\|x_{n}-z_{n}\right\|-\left(1+k_{n}\right) \alpha_{n}^{2}\left\|x_{n}-z_{n}-T^{n} z_{n}+T^{n} y_{n}\right\| \\
& -\alpha_{n}\left\|T^{n} x_{n+1}-T^{n} y_{n}-T^{n} z_{n+1}+T^{n} z_{n}\right\|-\left[\left(1+k_{n}\right) \alpha_{n} \gamma_{n}+\gamma_{n}\right]\left\|u_{n}-w_{n}\right\| \\
\geq & \left(1+\alpha_{n}\right)\left\|x_{n+1}-z_{n+1}\right\|
\end{aligned}
\end{aligned}
$$




$$
\begin{aligned}
& -\left[k_{n} \alpha_{n}+\left(1+k_{n}\right) \alpha_{n} \gamma_{n}+\gamma_{n}\right]\left\|x_{n}-z_{n}\right\|-\left(1+k_{n}\right) \alpha_{n}^{2}\left\|x_{n}-z_{n}-T^{n} z_{n}+T^{n} y_{n}\right\| \\
& -\alpha_{n}\left\|T^{n} x_{n+1}-T^{n} y_{n}-T^{n} z_{n+1}+T^{n} z_{n}\right\|-\left[\left(1+k_{n}\right) \alpha_{n} \gamma_{n}+\gamma_{n}\right]\left\|u_{n}-w_{n}\right\|,
\end{aligned}
$$

which implies that

$$
\begin{aligned}
\| x_{n+1} & -z_{n+1} \| \\
\leq & \left\{1+\left[\left(k_{n}-1\right) \alpha_{n}+\left(1+k_{n}\right) \alpha_{n} \gamma_{n}+\gamma_{n}\right]\right\}\left\|x_{n}-z_{n}\right\| \\
& +\left(1+k_{n}\right) \alpha_{n}^{2}\left\|x_{n}-z_{n}-T^{n} z_{n}+T^{n} y_{n}\right\|+\alpha_{n}\left\|T^{n} x_{n+1}-T^{n} y_{n}-T^{n} z_{n+1}+T^{n} z_{n}\right\| \\
& +\left[\left(1+k_{n}\right) \alpha_{n} \gamma_{n}+\gamma_{n}\right]\left\|u_{n}-w_{n}\right\| \\
\leq & \left\{1+\left[\left(k_{n}-1\right) \alpha_{n}+\left(1+k_{n}\right) \alpha_{n} \gamma_{n}+\gamma_{n}\right]\right\}\left\|x_{n}-z_{n}\right\| \\
& +\left(1+k_{n}\right) \alpha_{n}^{2}\left[\left\|x_{n}-z_{n}\right\|+\left\|T^{n} z_{n}-T^{n} y_{n}\right\|\right] \\
& +\alpha_{n}\left[\left\|T^{n} x_{n+1}-T^{n} y_{n}\right\|+\left\|T^{n} z_{n+1}-T^{n} z_{n}\right\|\right]+\left[\left(1+k_{n}\right) \alpha_{n} \gamma_{n}+\gamma_{n}\right]\left\|u_{n}-w_{n}\right\| \\
\leq & {\left[1+\left(k_{n}-1\right) \alpha_{n}+M \alpha_{n} \gamma_{n}+\gamma_{n}+M \alpha_{n}^{2}\right]\left\|x_{n}-z_{n}\right\|+M L \alpha_{n}^{2}\left\|z_{n}-y_{n}\right\| } \\
& +\alpha_{n} L\left(\left\|x_{n+1}-y_{n}\right\|+\left\|z_{n+1}-z_{n}\right\|\right)+2\left(M \alpha_{n} \gamma_{n}+\gamma_{n}\right) M .
\end{aligned}
$$

From (1.2) and (1.3), we obtain the following inequalities:

$$
\begin{aligned}
\| y_{n}- & z_{n} \| \\
= & \|\left(1-\beta_{n}-\delta_{n}\right)\left(x_{n}-z_{n}\right)+\beta_{n}\left(T^{n} x_{n}-T^{n} z_{n}\right) \\
& +\beta_{n}\left(T^{n} z_{n}-q\right)-\beta_{n}\left(z_{n}-q\right)+\delta_{n}\left(v_{n}-z_{n}\right) \| \\
\leq & \left(1+\beta_{n} L\right)\left\|x_{n}-z_{n}\right\|+\left(\beta_{n} L+\beta_{n}+\delta_{n}\right)\left\|z_{n}-q\right\|+\delta_{n}\left\|v_{n}-q\right\| \\
\leq & \left(1+\beta_{n} L\right)\left\|x_{n}-z_{n}\right\|+\left(\beta_{n} L+\beta_{n}+2 \delta_{n}\right) M \\
\left\|x_{n+1}-y_{n}\right\| & \\
= & \left\|\left(\beta_{n}+\delta_{n}-\alpha_{n}-\gamma_{n}\right) x_{n}+\alpha_{n} T^{n} y_{n}-\beta_{n} T^{n} x_{n}+\gamma_{n} u_{n}-\delta_{n} v_{n}\right\| \\
= & \|\left(\beta_{n}+\delta_{n}-\alpha_{n}-\gamma_{n}\right)\left(x_{n}-z_{n}\right)+\alpha_{n}\left(T^{n} y_{n}-T^{n} z_{n}\right)+\alpha_{n}\left(T^{n} z_{n}-q\right) \\
& -\alpha_{n}\left(z_{n}-q\right)-\beta_{n}\left(T^{n} x_{n}-T^{n} z_{n}\right)-\beta_{n}\left(T^{n} z_{n}-q\right)+\beta_{n}\left(z_{n}-q\right) \\
& -\gamma_{n}\left(z_{n}-q\right)+\delta_{n}\left(z_{n}-q\right)+\gamma_{n}\left(u_{n}-q\right)-\delta_{n}\left(v_{n}-q\right) \| \\
\leq & \left(\beta_{n}+\delta_{n}+\alpha_{n}+\gamma_{n}+\beta_{n} L\right)\left\|x_{n}-z_{n}\right\|+\alpha_{n} L\left\|y_{n}-z_{n}\right\| \\
& +\left[\left(\alpha_{n}+\beta_{n}\right)(L+1)+\left(\gamma_{n}+\delta_{n}\right)\right]\left\|z_{n}-q\right\|+\gamma_{n}\left\|u_{n}-q\right\|+\delta_{n}\left\|v_{n}-q\right\| \\
\leq & \left(\beta_{n}+\delta_{n}+\alpha_{n}+\gamma_{n}+\beta_{n} L\right)\left\|x_{n}-z_{n}\right\|+\alpha_{n} L\left\|y_{n}-z_{n}\right\| \\
& +\left[\left(\alpha_{n}+\beta_{n}\right)(L+1)+\left(2 \gamma_{n}+2 \delta_{n}\right)\right] M .
\end{aligned}
$$

Taking (2.6) into (2.7), we obtain that

$$
\begin{aligned}
\left\|x_{n+1}-y_{n}\right\| \leq & \left(\beta_{n}+\delta_{n}+\alpha_{n}+\gamma_{n}+\beta_{n} L\right)\left\|x_{n}-z_{n}\right\| \\
& +\alpha_{n} L\left\|y_{n}-z_{n}\right\|+\left[\left(\alpha_{n}+\beta_{n}\right)(L+1)+\left(2 \gamma_{n}+2 \delta_{n}\right)\right] M
\end{aligned}
$$




$$
\begin{aligned}
\leq & \left(\beta_{n}+\delta_{n}+\alpha_{n}+\gamma_{n}+\beta_{n} L\right)\left\|x_{n}-z_{n}\right\| \\
& +\alpha_{n} L\left[\left(1+\beta_{n} L\right)\left\|x_{n}-z_{n}\right\|+\left(\beta_{n} L+\beta_{n}+2 \delta_{n}\right) M\right] \\
& +\left[\left(\alpha_{n}+\beta_{n}\right)(L+1)+\left(2 \gamma_{n}+2 \delta_{n}\right)\right] M \\
\leq & {\left[\beta_{n}+\delta_{n}+\alpha_{n}(L+1)+\gamma_{n}+\beta_{n} L+\alpha_{n} \beta_{n} L^{2}\right]\left\|x_{n}-z_{n}\right\| } \\
& +\left[\left(\alpha_{n}+\beta_{n}\right)(L+1)+\left(2 \gamma_{n}+2 \delta_{n}\right)+\alpha_{n} L\left(\beta_{n} L+\beta_{n}+2 \delta_{n}\right)\right] M .
\end{aligned}
$$

From (1.3), we get

$$
\begin{aligned}
\left\|z_{n+1}-z_{n}\right\| & =\left\|\alpha_{n}\left(T^{n} z_{n}-q\right)+\gamma_{n}\left(w_{n}-q\right)-\left(\alpha_{n}+\gamma_{n}\right)\left(z_{n}-q\right)\right\| \\
& \leq \alpha_{n}\left\|T^{n} z_{n}-q\right\|+\gamma_{n}\left\|w_{n}-q\right\|+\left(\alpha_{n}+\gamma_{n}\right)\left\|z_{n}-q\right\| \\
& \leq\left(\alpha_{n}+\alpha_{n} L+\gamma_{n}\right)\left\|z_{n}-q\right\|+\gamma_{n}\left\|w_{n}-q\right\| \\
& \leq\left(\alpha_{n}+\alpha_{n} L+2 \gamma_{n}\right) M .
\end{aligned}
$$

Substituting (2.6), (2.8) and (2.9) into (2.5), we have

$$
\left\|x_{n+1}-z_{n+1}\right\| \leq\left(1+B_{n}\right)\left\|x_{n}-z_{n}\right\|+C_{n}
$$

where $B_{n}=\left(k_{n}-1\right) \alpha_{n}+M \alpha_{n} \gamma_{n}+\gamma_{n}+M \alpha_{n}^{2}+M L \alpha_{n}^{2}\left(1+\beta_{n} L\right)+\alpha_{n} L\left[\beta_{n}+\delta_{n}+\alpha_{n}(L+1)+\right.$ $\left.\gamma_{n}+\beta_{n} L+\alpha_{n} \beta_{n} L^{2}\right], C_{n}=M^{2} L \alpha_{n}^{2}\left(\beta_{n} L+\beta_{n}+2 \delta_{n}\right)+M L\left(\alpha_{n}^{2}+\alpha_{n}^{2} L+2 \alpha_{n} \gamma_{n}\right)+2\left(M \alpha_{n} \gamma_{n}+\right.$ $\left.\gamma_{n}\right) M+\alpha_{n} M L\left[\left(\alpha_{n}+\beta_{n}\right)(L+1)+\left(2 \gamma_{n}+2 \delta_{n}\right)+\alpha_{n} L\left(\beta_{n} L+\beta_{n}+2 \delta_{n}\right)\right]$ and satisfy $\sum_{n=0}^{\infty} B_{n}<\infty$, $\sum_{n=0}^{\infty} C_{n}<\infty$. By Lemma 1.11, $\lim _{n \rightarrow \infty}\left\|x_{n}-z_{n}\right\|$ exists. Hence the sequence $\left\{x_{n}-z_{n}\right\}$ is bounded. Set $M_{1}=\sup _{n}\left\{\left\|x_{n}-z_{n}\right\|\right\}$.

It follows from (1.2), (1.3), (2.1) and Lemma 1.10 that we have

$$
\begin{aligned}
\| x_{n+1} & -z_{n+1} \|^{2} \\
= & \left\|\left(1-\alpha_{n}-\gamma_{n}\right)\left(x_{n}-z_{n}\right)+\alpha_{n}\left(T^{n} y_{n}-T^{n} z_{n}\right)+\gamma_{n}\left(u_{n}-w_{n}\right)\right\|^{2} \\
\leq & \left(1-\alpha_{n}\right)^{2}\left\|x_{n}-z_{n}\right\|^{2}+2 \alpha_{n}\left\langle T^{n} x_{n+1}-T^{n} z_{n+1}, j\left(x_{n+1}-z_{n+1}\right)\right\rangle \\
& +2 \alpha_{n}\left\langle T^{n} y_{n}-T^{n} z_{n}-T^{n} x_{n+1}+T^{n} z_{n+1}, j\left(x_{n+1}-z_{n+1}\right)\right\rangle+2 M \gamma_{n}\left\|x_{n+1}-z_{n+1}\right\| \\
\leq & \left(1-\alpha_{n}\right)^{2}\left\|x_{n}-z_{n}\right\|^{2}+2 \alpha_{n}\left[k_{n}\left\|x_{n+1}-z_{n+1}\right\|^{2}-\sigma\left(\left\|x_{n+1}-z_{n+1}\right\|\right)\right] \\
& +2 \alpha_{n} L\left(\left\|x_{n+1}-y_{n}\right\|+\left\|z_{n+1}-z_{n}\right\|\right) \cdot\left\|x_{n+1}-z_{n+1}\right\|+2 M M_{1} \gamma_{n} \\
\leq & \left(1-\alpha_{n}\right)^{2}\left\|x_{n}-z_{n}\right\|^{2}+2 \alpha_{n} k_{n}\left\|x_{n+1}-z_{n+1}\right\|^{2}-2 \alpha_{n} \sigma\left(\left\|x_{n+1}-z_{n+1}\right\|\right) \\
& +E_{n}+2 L M_{1} \alpha_{n}\left\|z_{n+1}-z_{n}\right\|,
\end{aligned}
$$

where $E_{n}=2 L M_{1}^{2} \alpha_{n}\left[\beta_{n}+\delta_{n}+\alpha_{n}(L+1)+\gamma_{n}+\beta_{n} L+\alpha_{n} \beta_{n} L^{2}\right]+2 L M_{1} M\left[\left(\alpha_{n}+\beta_{n}\right)(L+1)+\right.$ $\left.2 \gamma_{n}+2 \delta_{n}+\alpha_{n} L\left(\beta_{n} L+\beta_{n}+2 \delta_{n}\right)\right]+2 M M_{1} \gamma_{n}$. Since $\lim _{n \rightarrow \infty}\left(1-2 k_{n} \alpha_{n}\right)=1$, then there exists $N_{3}$ such that $1>1-2 k_{n} \alpha_{n}>\frac{1}{2}$ for $n>N_{3}$. So (2.11) becomes

$$
\begin{aligned}
& \left\|x_{n+1}-z_{n+1}\right\|^{2} \\
& \quad \leq \frac{\left(1-\alpha_{n}\right)^{2}}{1-2 \alpha_{n} k_{n}}\left\|x_{n}-z_{n}\right\|^{2}-2 \alpha_{n} \sigma\left(\left\|x_{n+1}-z_{n+1}\right\|\right)+2 E_{n}+4 L M_{1} \alpha_{n}\left\|z_{n+1}-z_{n}\right\|
\end{aligned}
$$




$$
\begin{aligned}
\leq & {\left[1+4\left(k_{n}-1\right) \alpha_{n}\right]\left\|x_{n}-z_{n}\right\|^{2}-2 \alpha_{n} \sigma\left(\left\|x_{n+1}-z_{n+1}\right\|\right) } \\
& +2 E_{n}+2 M_{1} \alpha_{n}^{2}+4 L M_{1} \alpha_{n}\left\|z_{n+1}-z_{n}\right\| .
\end{aligned}
$$

Since $\lim _{n \rightarrow \infty} k_{n}=1, \lim _{n \rightarrow \infty}\left\|z_{n}-q\right\|=0$, then $\left(k_{n}-1\right) \alpha_{n}=o\left(\alpha_{n}\right), L M_{1} \alpha_{n}\left\|z_{n+1}-z_{n}\right\|=$ $o\left(\alpha_{n}\right)$. By (iii) and (iv), we have $\sum_{n=0}^{\infty}\left(E_{n}+\alpha_{n}^{2}\right)<\infty$. Using Lemma 1.12, we obtain that $\lim _{n \rightarrow \infty}\left\|x_{n}-z_{n}\right\|=0$.

Theorem 2.2 Let D be a nonempty closed convex subset of the real Banach space E. Suppose that $T: D \longrightarrow D$ is a uniformly L-Lipschitz asymptotically pseudocontractive mapping with the real number sequence $\left\{k_{n}\right\} \subset[1,+\infty), \lim _{n \rightarrow \infty} k_{n}=1$. Suppose that $\left\{x_{n}\right\}$ is defined by (1.2), where $\left\{\alpha_{n}\right\},\left\{\gamma_{n}\right\},\left\{\beta_{n}\right\},\left\{\delta_{n}\right\}$ are four real number sequences in $[0,1]$ satisfying the following conditions:

(i) $\alpha_{n}+\gamma_{n} \leq 1, \beta_{n}+\delta_{n} \leq 1$

(ii) $\lim _{n \rightarrow \infty} \alpha_{n}=0, \sum_{n=0}^{\infty} \alpha_{n}=\infty$;

(iii) $\sum_{n=0}^{\infty} \alpha_{n}^{2}<\infty, \sum_{n=0}^{\infty} \gamma_{n}<\infty$;

(iv) $\sum_{n=0}^{\infty} \alpha_{n}\left(\beta_{n}+\delta_{n}\right)<\infty, \sum_{n=0}^{\infty} \alpha_{n}\left(k_{n}-1\right)<\infty$.

Suppose $q \in F(T) \neq \emptyset$. If there exists a strictly increasing continuous function $\Phi:[0,+\infty) \rightarrow$ $[0,+\infty)$ with $\Phi(0)=0$ such that

$$
\left\langle T^{n} x_{n+1}-q, j\left(x_{n+1}-q\right)\right\rangle \leq k_{n}\left\|x_{n+1}-q\right\|^{2}-\sigma\left(\left\|x_{n+1}-q\right\|\right), \quad \forall n \geq 0,
$$

where $\sigma(t)=\frac{\Phi(t)}{1+\Phi(t)+t^{2}}$, then the modified Ishikawa iteration with errors $\left\{x_{n}\right\}$ converges strongly to $q \in F(T)$.

Proof In the proof course of Theorem 2.1, setting $z_{n}=q$, for $\forall n \geq 1$, we obtain Theorem 2.2.

It is worth mentioning that the result extends Theorem 3.1 in [8] by dropping the bounded assumption. See the following example.

Example 2.3 Let $E=R$ be a real space with the usual norm. Define $T: E \rightarrow E$ by $T x=\frac{2}{3} x$, $\Phi(t)=t^{4}, t \in[0,+\infty), k_{n}=1, \forall n \geq 1$. Then $\Phi$ is a strictly increasing continuous function with $\Phi(0)=0$ and $T$ has a fixed point $q=0$. For any $x, y \in E$, we obtain that

$$
\begin{aligned}
& \left\langle T^{n} x-T^{n} y, j(x-y)\right\rangle \\
& =\left(\frac{2}{3}\right)^{n}\|x-y\|^{2} \\
& \quad \leq \frac{2}{3}\|x-y\|^{2} \\
& \quad \leq\|x-y\|^{2}-\frac{\|x-y\|^{4}}{1+\|x-y\|^{4}+\|x-y\|^{2}} \\
& =\|x-y\|^{2}-\frac{\Phi(\|x-y\|)}{1+\Phi(\|x-y\|)+\|x-y\|^{2}} \\
& =k_{n}\|x-y\|^{2}-\sigma(\|x-y\|), \quad \forall n \geq 1 .
\end{aligned}
$$

Then the mapping $T$ satisfies Theorem 2.2. But the range of $T$ is not bounded. 
Corollary 2.4 Let D be a nonempty closed convex subset of the real Banach space E. Suppose that $T: D \longrightarrow D$ is a uniformly L-Lipschitz asymptotically pseudocontractive mapping with the real number sequence $\left\{k_{n}\right\} \subset[1,+\infty), \lim _{n \rightarrow \infty} k_{n}=1$. Let $\left\{x_{n}\right\}$ be defined by (1.3), where $\left\{\alpha_{n}\right\},\left\{\beta_{n}\right\}$ are two real number sequences in $[0,1]$ satisfying the following conditions:

(i) $\lim _{n \rightarrow \infty} \alpha_{n}=0, \sum_{n=0}^{\infty} \alpha_{n}=\infty$;

(ii) $\sum_{n=0}^{\infty} \alpha_{n}^{2}<\infty, \sum_{n=0}^{\infty} \alpha_{n}\left(k_{n}-1\right)<\infty$;

(iii) $\sum_{n=0}^{\infty} \alpha_{n} \beta_{n}<\infty$.

Suppose $q \in F(T) \neq \emptyset$. If there exists a strictly increasing continuous function $\Phi:[0,+\infty) \rightarrow$ $[0,+\infty)$ with $\Phi(0)=0$ such that

$$
\left\langle T^{n} x_{n+1}-q, j\left(x_{n+1}-q\right)\right\rangle \leq k_{n}\left\|x_{n+1}-q\right\|^{2}-\sigma\left(\left\|x_{n+1}-q\right\|\right), \quad \forall n \geq 1,
$$

where $\sigma(t)=\frac{\Phi(t)}{1+\Phi(t)+t^{2}}$, then the modified Mann iteration $\left\{u_{n}\right\}$ converges strongly to $q \in F(T)$.

Proof In Theorem 2.2, setting $\gamma_{n}=\delta_{n}=0$, we obtain Corollary 2.4.

The control conditions of the parameters in Corollary 2.4 are different from those of Theorem 2.1 of [6]. See the following example.

Example 2.5 Set $\alpha_{n}=\frac{1}{\sqrt{n}}, \beta_{n}=\frac{1}{\sqrt{n}}, k_{n}=1+\frac{1}{\sqrt{n}}, \forall n \geq 1$. Then $\alpha_{n}, \beta_{n} \rightarrow 0$ as $n \rightarrow \infty$ and $\sum_{n=0}^{\infty} \alpha_{n}=\infty$, but $\sum_{n=0}^{\infty} \alpha_{n}^{2}=\infty, \sum_{n=0}^{\infty} \alpha_{n} \beta_{n}=\infty$ and $\sum_{n=0}^{\infty} \alpha_{n}\left(k_{n}-1\right)=\infty$. On the other hand, let

$$
\alpha_{n}=\left\{\begin{array}{ll}
0, & n=2 i-1, \\
\frac{1}{2 i}, & n=2 i,
\end{array} \quad \beta_{n}=\left\{\begin{array}{ll}
2 i-1, & n=2 i-1, \\
\frac{1}{2 i}, & n=2 i,
\end{array} \quad k_{n}=1+\frac{1}{n}, \quad \forall i \geq 1, n \geq 1 .\right.\right.
$$

Then $\alpha_{n} \rightarrow 0$ as $n \rightarrow \infty, \sum_{n=0}^{\infty} \alpha_{n}=\infty$ and $\sum_{n=0}^{\infty} \alpha_{n}^{2}<\infty, \sum_{n=0}^{\infty} \alpha_{n} \beta_{n}<\infty, \sum_{n=0}^{\infty} \alpha_{n}\left(k_{n}-\right.$ 1) $<\infty$, but $\beta_{n} \rightarrow 0$ as $n \rightarrow \infty$ does not hold.

Remark 2.6 Our theorems extend and improve the corresponding results of $[2,6-8]$ in the following sense:

(1) We point out the problems of [2, 6, 7] and revise them.

(2) We remove the hypothesis $T$ with bounded range and obtain the same result by the different method from [8].

(3) We extend formula (2.1) of [8] to (2.13) in this paper.

(4) We also obtain the equivalence between the convergence of the modified Mann iteration with errors and the modified Ishikawa iteration with errors for an asymptotically pseudocontractive mapping.

\section{Competing interests}

The authors declare that they have no competing interests.

Authors' contributions

All the authors completed this paper together. They all read and approved the final manuscript.

\section{Acknowledgements}

The authors acknowledge the financial support of the National Natural Science Foundation of China (Grant No.

11372196) and the Shijiazhuang Tiedao University Foundation (Grant No. 20133026).

Received: 12 August 2013 Accepted: 2 July 2014 Published: 18 August 2014 


\section{References}

1. Chang, SS: Iterative approximation problem of fixed points for asymptotically nonexpansive mappings in Banach spaces. Acta Math. Appl. Sin. 24(2), 236-241 (2001) (in Chinese)

2. Rhoades, BE, Soltuz, SM: The equivalence between the convergence of Ishikawa and Mann iterations for an asymptotically pseudocontractive map. J. Math. Anal. Appl. 283, 681-688 (2003)

3. Schu, J: Iterative construction of fixed point of asymptotically nonexpansive mappings. J. Math. Anal. Appl. 158 407-413 (1991)

4. Ishikawa, S: Fixed point by a new iteration method. Proc. Am. Math. Soc. 44, 147-150 (1974)

5. Mann, WR: Mean value in iteration. Proc. Am. Math. Soc. 4, 506-510 (1953)

6. Xue, ZQ: The convergence theorem for common fixed point of uniformly L-Lipschitzian asymptotically $\Phi$-pseudocontractive mappings. Bull. Korean Math. Soc. 47(2), 295-305 (2010)

7. Xue, ZQ: The equivalence among the modified Mann-Ishikawa and Noor iterations for uniformly L-Lipschitzian mappings in Banach spaces. J. Math. Inequal. 4(3), 345-354 (2010)

8. Zeng, LC: Modified Ishikawa iterative process with errors in Banach spaces. Acta Math. Sin. 47(2), $219-228$ (2004) (in Chinese)

9. Ofoedu, EU: Strongly convergence theorem for uniformly L-Lipschitzian asymptotically pseudocontractive mapping in real Banach space. J. Math. Anal. Appl. 321(2), 722-728 (2006)

10. Osilike, MO, Aniagbosor, SC: Weak and strong convergence theorem for fixed points of asymptotically nonexpansive mapping. Math. Comput. Model. 32(10), 1181-1191 (2000)

doi:10.1186/1029-242X-2014-293

Cite this article as: Lv and Xue: The equivalence between the convergence of the modified Mann and Ishikawa iterations for asymptotically pseudocontractive mappings obtained by dropping the bounded assumption. Journal of Inequalities and Applications 2014 2014:293.

\section{Submit your manuscript to a SpringerOpen ${ }^{\ominus}$ journal and benefit from:}

- Convenient online submission

- Rigorous peer review

- Immediate publication on acceptance

- Open access: articles freely available online

- High visibility within the field

- Retaining the copyright to your article 